\title{
Comment on "Cervical pedicle screw instrumentation is reliable with 0-arm-based 3D navigation: analysis of cervical pedicle screw placement accuracy with 0-arm-based 3D navigation" by S. Chachan et al. (Eur Spine J; 2018: doi: 10.1007/s00586-018-5585-1)
}

\author{
R. Cecchinato ${ }^{1} \cdot$ C. Lamartina $^{1}$
}

Received: 13 May 2018 / Accepted: 11 June 2018 / Published online: 19 June 2018

c) Springer-Verlag GmbH Germany, part of Springer Nature 2018

The paper entitled "Cervical pedicle screw instrumentation is reliable with $\mathrm{O}$-arm-based 3D navigation: analysis of cervical pedicle screw placement accuracy with O-armbased 3D navigation" raises some questions regarding the relation between risks and benefits derived from surgical techniques. It is a well-structured paper on the effectiveness of O-arm helping surgeons in implanting cervical pedicle screws. Authors found a $92.95 \%$ accuracy rate, based on the analysis of 241 pedicle screws implanted between $\mathrm{C} 2$ and C7. No neurovascular complications were observed consequent to malpositioned screws. Even if no control group is present, it is easily understandable how a navigation support can be really helpful in this kind of instrumentation. Even if rare clinical consequences have been described in different published studies, a rate of $7-15 \%$ pedicle breeches can be observed due to the small size of cervical pedicles. Complications of misplaced hardware at this level can have devastating consequences, with possible cord injury, nerve roots damage or vascular lesions. In 2000, a study from Abumi et al. analyzed 712 cervical pedicle screws, with one case of vertebral artery injury and two cases of radiculopathy given by a conflict between a nerve root and a malpositioned screw. This is why a comparison between these risks and the possible benefits of this surgical technique is recommended. There are no doubts regarding the improved stability of cervical pedicle instrumentation compared to lateral masses. In 2006, Johnston et al. found that cervical pedicle screws loosened less than lateral mass screws in a cyclic uniplanar fatigue model, with higher pullout strengths related to screw insertion torque and specimen bone density. In a subsequent study, the stability of pedicle screw constructs was found superior to lateral masses one in a simulation of a type $\mathrm{C}$ injury in a bovine model. The mean load-to-failure was found to be $677 \mathrm{~N}$ for cervical pedicle screws, versus $355 \mathrm{~N}$ for lateral masses screws. Moreover, the diameter of pedicle screws does not seem to affect the pullout values, since similar pullout strengths have been observed between 2.7- and 3.5-mm screws.

The translation of these theoretical advantages on human application is the discussion point. Which are the cases where cervical pedicle screws should be strongly recommended (in a paper from 2015 we proposed their use in B-type cervical injuries)? Where their biomechanical features represent a real advantage for patients? Are the different instrumentation techniques able to affect in different ways the alignment of the cervical spine? The answers to these questions should represent the goal of future studies on this topic. Multicentric long-term follow-up study designs should be considered, and registries may help in collecting data on revision and complication rates. Life span of the different techniques should be compared in different pathologies, to determine the long-term effectiveness. When specific indications and recommendations for cervical pedicle screws implantation will be defined, CT-based or mechanical navigation systems will surely be recommended in this challenging procedure.

R. Cecchinato

dott.cecchinato@gmail.com

1 GSpine4, IRCCS Istituto Ortopedico Galeazzi, Via Riccardo Galeazzi, 4, 20161 Milan, Italy 